Document downloaded from:

http://hdl.handle.net/10251/50793

This paper must be cited as:

Reig, J. (2008). Performance of Maximal Ratio Combiners over Correlated Nakagami-m Fading Channels with Arbitrary Fading Parameters. IEEE Transactions on Wireless Communications. 7(5):1441-1445. doi:10.1109/TWC.2008.060129.

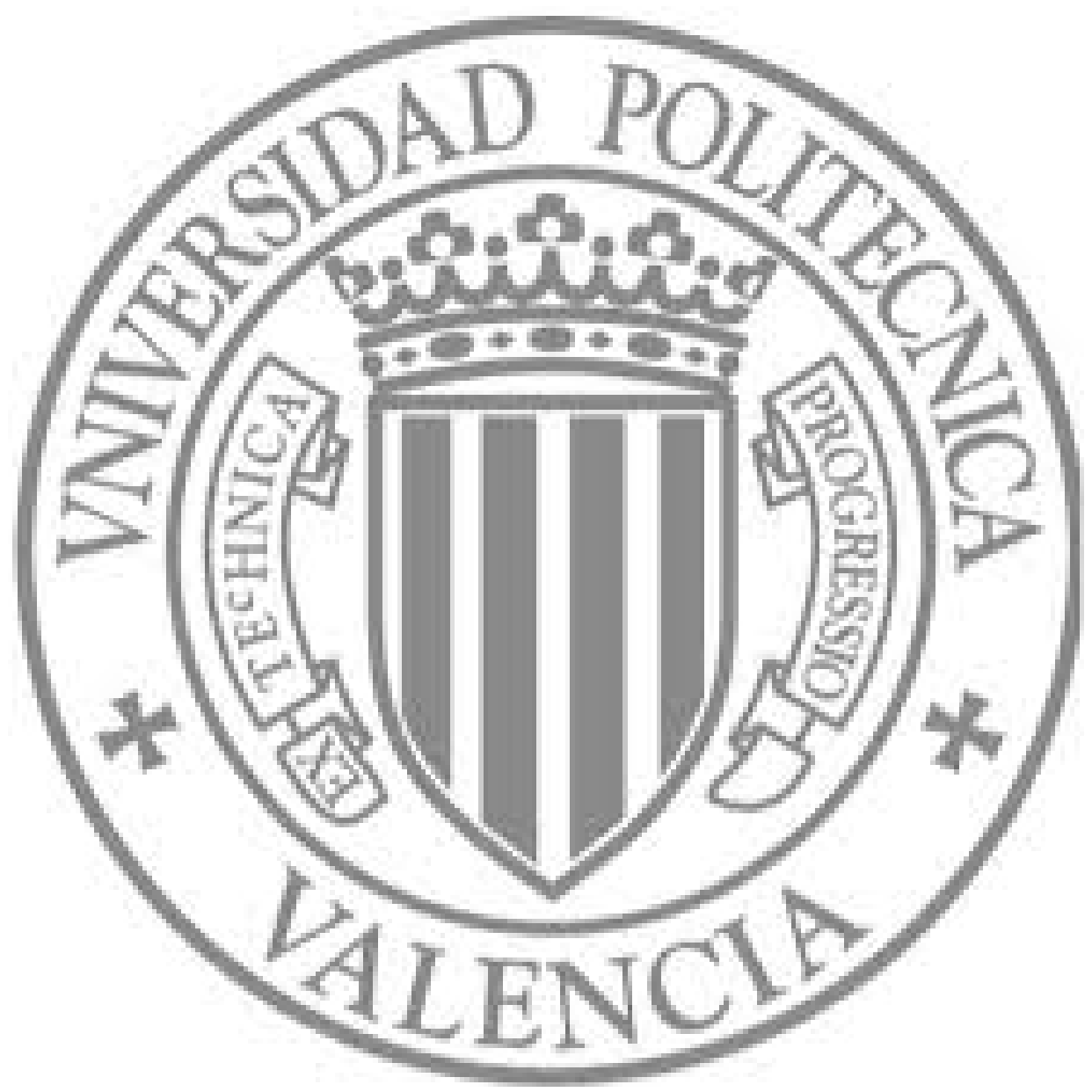

The final publication is available at

http://dx.doi.org/10.1109/TWC.2008.060129

Copyright Institute of Electrical and Electronics Engineers (IEEE) 


\title{
Performance of Maximal Ratio Combiners over Correlated Nakagami- $m$ Fading Channels with Arbitrary Fading Parameters
}

\author{
Juan Reig, Member, IEEE
}

\section{Keywords}

Gamma distributions, fading channels, probability, correlation, land mobile radio cellular systems.

\begin{abstract}
In this letter, performance metrics of maximal ratio combiners (MRC) over correlated Nakagami-m fading are calculated with both arbitrary fading parameters and average powers. We derive the moment generating function $(M G F)$ of the sum of correlated gamma variables with arbitrary fading parameters. Using the MGF-based approach, we obtain the variance of the signal-to-noise ratio (SNR) at the output of the combiner, the outage probability, the average symbol error rate for coherent multichannel reception, and the diversity gain. The results for an exponentially decaying model of the fading parameter are presented and discussed.
\end{abstract}

Dr. Reig is with the iTEAM, Departament of Communications, Technical University of Valencia, 46022 Valencia, Spain. E-mail: jreigp@dcom.upv.es. Ph.: +34 963879762 


\section{INTRODUCTION}

The Nakagami- $m$ distribution [1] is a usual model to describe the envelope of the received signal in cellular land mobile and indoor mobile radio systems due to its versatility and accuracy in matching experimental data. Furthermore, this distribution includes the classical Rayleigh model as a special case.

The distribution of the signal-to-noise ratio (SNR) at a maximal-ratio combiner (MRC) output with correlated Nakagami- $m$ fading can be expressed as the sum of correlated gamma variables.

Previous works assumed that the fading parameters of each distribution were identical [2]-[5]. Nevertheless, there are some examples of diversity systems, like angle or polarization diversity, or the RAKE receivers, where the average power and the fading parameter are not necessarily equal for each branch.

One step beyond, Win et al. [6] have developed an analytical framework to study the performance of wireless systems using MRC with an arbitrary number of branches considering coherent detection of the received signals, where SNRs can be arbitrarily correlated, and the fading parameters and the average SNRs of the branches are not necessarily equal. Nevertheless, the double of the fading parameter of each branch signal was restricted to integer values.

Recently, Aalo et al. [7] obtained the probability density function (PDF) of the SNR at the output of the combiner, the outage probability and the average probability of error of MRC schemes in Nakagami- $m$ channels. The fading parameters and the average SNR at each input of the combiner were assumed arbitrary. This work assumes total independence between fading signals at each input of the combiner.

On the other hand, Gifford et al. [8] studied the MRC diversity performance in the presence of non-ideal channel estimates.

In [9]-[11], the performance of hybrid selection/maximal-ratio combining (HS/MRC) diversity system over Rayleigh and Nakagami- $m$ channels was analyzed. 
More recently, in [12], the average probability of error of a two-dimensional (2D) RAKE receiver over correlated Nakagami- $m$ fading with spatial correlation was obtained.

In this letter, the derivation of the moment generating function (MGF) of the SNR at the output of a MRC in a correlated Nakagami- $m$ fading is presented. The fading parameters at each combiner input signal are arbitrary, not necessarily integer. Using the MGF, the average symbol error probability (ASEP) for several modulations, the probability of outage and the variance of the SNR at the output of the combiner are evaluated.

II. MGF OF THE MULTIVARIATE GAMMA DISTRIBUTION WITH ARBRITRARY FADING PARAMETERS

Let $s_{1}, \ldots, s_{N}$ be gamma variables calculated from

$$
\begin{aligned}
& s_{1}=s_{1 a}+s_{2 b}=\sum_{k=1}^{m_{N}} r_{1, k}^{2}+\sum_{l=m_{N}+1}^{m_{1}} r_{1, l}^{2} \\
& s_{2}=s_{2 a}+s_{2 b}=\sum_{k=1}^{m_{N}} r_{2, k}^{2}+\sum_{l=m_{N}+1}^{m_{2}} r_{2, l}^{2} \\
& s_{3}=s_{3 a}+s_{3 b}=\sum_{k=1}^{m_{N}} r_{3, k}^{2}+\sum_{l=m_{N}+1}^{m_{3}} r_{3, l}^{2}, \\
& \ldots \\
& s_{N}=s_{N a}=\sum_{k=1}^{m_{N}} r_{N, k}^{2}
\end{aligned}
$$

where $r_{1, i_{1}}^{2}, \ldots, r_{N, i_{M}}^{2}, i_{n}=1, \ldots, m_{n}, n=1, \ldots, N$ are exponentially distributed random variables that are mutually independent for $i_{u} \neq i_{v}, \quad u, v=1, \ldots, N$ with $\overline{r_{1, i_{1}}^{2}}=\Omega_{1}, \ldots, \overline{r_{N, i_{N}}^{2}}=\Omega_{N}$, respectively, where - denotes expectation. The variables $s_{n a}=\sum_{k=1}^{m_{N}} r_{n, k}^{2}, s_{n b}=\sum_{l=m_{N}+1}^{m_{n}} r_{n, l}^{2}$ are subject to $m_{1} \geq m_{2} \geq \ldots \geq m_{N-1} \geq m_{N}$, which are integer values, and the correlation coefficient between $r_{p, u}^{2}$ and $r_{q, u}^{2}, u=1, . ., N$ is given by 


$$
k_{p q}=\left\{\begin{array}{cc}
1 & p=q \\
\frac{\overline{r_{p, u}^{2} \cdot r_{q, u}^{2}}-\overline{r_{p, u}^{2}} \cdot \overline{r_{q, u}^{2}}}{\sqrt{\sigma_{r_{p, u}^{2}}^{2} \cdot \sigma_{r_{q, u}^{2}}^{2}}} & p \neq q
\end{array}\right.
$$

where $\sigma_{r_{p, u}^{2}}^{2}$ and $\sigma_{r_{q, u}^{2}}^{2}$ are the variances of $r_{p, u}^{2}$ and $r_{q, u}^{2}$, respectively.

Hence, $s_{1 a}, s_{2 a}, \ldots, s_{N a}$ follow gamma distributions whose PDF is given by

$$
p_{s_{n a}}\left(s_{n a}\right)=\frac{1}{\Gamma\left(m_{N}\right)} \frac{s_{n a}^{m_{N}-1}}{\Omega_{n}^{m_{N}}} \exp \left(-\frac{s_{n a}}{\Omega_{n}}\right), \quad n=1, \ldots, N,
$$

where

$$
\overline{s_{n a}}=\frac{\Omega_{n}}{m_{N}}, \quad m_{N}=\frac{{\overline{s_{n a}}}^{2}}{\overline{\left(s_{n a}-\overline{s_{n a}}\right)^{2}}} .
$$

The MGF of the multivariate gamma distribution for $s_{1 a}, s_{2 a}, \ldots, s_{N a}$ with identical fading parameters can be evaluated from [13] as

$$
\begin{aligned}
& \mathcal{M}_{s_{1 a}, \ldots, s_{N a}}\left(t_{1 a}, \ldots, t_{N a}\right)=\prod_{n=1}^{N}\left(1-t_{n a} \cdot \Omega_{n}\right)^{-m_{N}}
\end{aligned}
$$

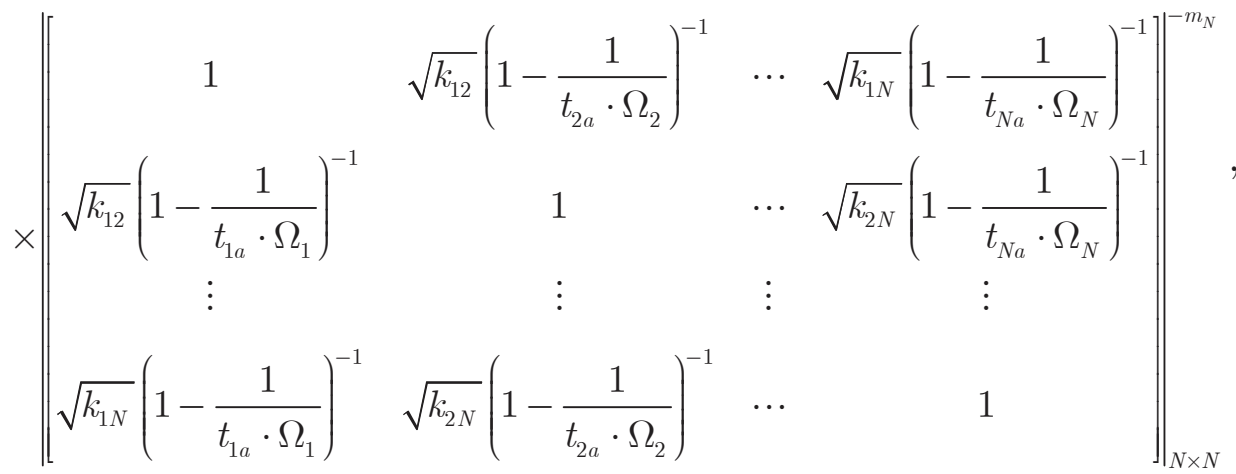

where $|\cdot|$ is the determinant operator.

From $(1), s_{1}, s_{2}, \ldots, s_{N}$ are gamma variables with parameters

$$
\overline{s_{n}}=\frac{\Omega_{n}}{m_{n}}, \quad m_{n}=\frac{{\overline{s_{n}}}^{2}}{\overline{\left(s_{n}-\overline{s_{n}}\right)^{2}}} .
$$

Using an elemental transformation of variables $\varepsilon_{1}=\frac{s_{1}}{m_{1}}, \ldots, \varepsilon_{N}=\frac{s_{N}}{m_{N}}$, the MGF of $\varepsilon_{1}, \ldots, \varepsilon_{N}$ can be constructed as 


$$
\begin{aligned}
& \mathcal{M}_{\varepsilon_{1}, \ldots, \varepsilon_{N}}\left(t_{1}, \ldots, t_{N}\right)=\prod_{n=1}^{N}\left(1-\frac{t_{n} \cdot \Omega_{n}}{m_{n}}\right)^{-m_{n}} \\
& \times\left\|\begin{array}{cccc}
1 & \sqrt{k_{12}}\left(1-\frac{m_{N}}{t_{2} \cdot \Omega_{2}}\right)^{-1} & \cdots & \sqrt{k_{1 N}}\left(1-\frac{m_{N}}{t_{N} \cdot \Omega_{N}}\right)^{-1} \\
\sqrt{k_{12}}\left(1-\frac{m_{N}}{t_{1} \cdot \Omega_{1}}\right)^{-1} & 1 & \cdots & \sqrt{k_{2 N}}\left(1-\frac{m_{N}}{t_{N} \cdot \Omega_{N}}\right)^{-1} \\
\vdots & \vdots & \vdots & \vdots \\
\sqrt{k_{1 N}}\left(1-\frac{m_{N}}{t_{1} \cdot \Omega_{1}}\right)^{-1} & \sqrt{k_{2 N}}\left(1-\frac{m_{N}}{t_{2} \cdot \Omega_{2}}\right)^{-1} & \cdots & 1
\end{array}\right\|_{N \times N}
\end{aligned}
$$

The MGF given by (7) is consistent for $m_{1}, \ldots, m_{N}$ non-integer values in the same way as the derivation of the characteristic function for the bivariate gamma distribution of $[1,(123)]$.

From (1) and applying the definition of (2), the correlation coefficient between $\varepsilon_{p}$ and $\varepsilon_{q}$ can be written as

$$
\rho_{p q}=k_{p q} \frac{m_{N}}{\sqrt{m_{p} \cdot m_{q}}}, \quad p, q=1, \ldots, N, \quad p<q .
$$

Obviously, the distribution of $\varepsilon_{1}, \ldots, \varepsilon_{N}$, whose MGF is given by (7), is subject to

$$
k_{p q}=\frac{\rho_{p q} \cdot \sqrt{m_{p} \cdot m_{q}}}{m_{N}}<1, \quad p, q=1, \ldots, N, \quad p<q .
$$

For $N=2$, the MGF of $\varepsilon_{1}, \varepsilon_{2}$ is given by

$$
\begin{aligned}
& \mathcal{M}_{\varepsilon_{1}, \varepsilon_{2}}\left(t_{1}, t_{2}\right)=\left(\frac{m_{1}}{\Omega_{1}}\right)^{m_{1}}\left(\frac{m_{2}}{\Omega_{2}}\right)^{m_{2}} \frac{1}{\left(1-\rho_{12} \sqrt{m_{1} / m_{2}}\right)^{m_{2}}} \frac{1}{\left(\frac{m_{1}}{\Omega_{1}}-t_{1}\right)^{m_{1}-m_{2}}} \\
& 1 \\
& \overline{\left(\left(\frac{m_{1}}{\Omega_{1}\left(1-\rho_{12} \sqrt{m_{1} / m_{2}}\right)}-t_{1}\right)\left(\frac{m_{2}}{\Omega_{2}\left(1-\rho_{12} \sqrt{m_{1} / m_{2}}\right)}-t_{2}\right)-\frac{m_{1} m_{2} \rho_{12} \sqrt{m_{1} / m_{2}}}{\Omega_{1} \Omega_{2}\left(1-\rho_{12} \sqrt{m_{1} / m_{2}}\right)^{2}}\right)^{m_{2}}}
\end{aligned}
$$

which corresponds to the characteristic function of the bivariate gamma distribution with arbitrary fading parameters given in $[14,(4)]$, substituting $\Omega_{1} / m_{1}, \Omega_{2} / m_{2}$ for the average powers of the marginal distributions and $-t_{1},-t_{2}$ for the variables of the characteristic function, respectively. This equation is subject to $m_{1} \geq m_{2}$. 
Nevertheless, in urban wireless environments, the fading parameters typically oscillate in the range from 1 to 2.5 [15]. Therefore, we could use the MGF given by $(7)$ to achieve high correlation coefficients, $\rho_{p q}$, in such scenarios.

Hence, the MGF of the sum of correlated gamma variates with arbitrary fading parameters, $\varepsilon=\varepsilon_{1}+\ldots+\varepsilon_{N}$ can be written as

$$
\mathcal{M}_{\varepsilon}(t)=\mathcal{M}_{\varepsilon_{1}, \ldots, \varepsilon_{N}}\left(t_{1}=t, \ldots, t_{N}=t\right),
$$

where $\mathcal{M}_{\varepsilon_{1}, \ldots, \varepsilon_{N}}\left(t_{1}, \ldots, t_{N}\right)$ is given by $(7)$.

\section{PERFORMANCE ANALYSIS OF MRC}

For MRC and post-detection equal-gain combining (or square law combining as it is also called [16, p. 298]), the conditional total SNR per symbol, $\gamma$, can be calculated as

$$
\gamma=\sum_{i=1}^{N} \gamma_{i}
$$

where $\gamma_{i}=r_{i}^{2} E_{S} / N_{0}(i=1, \ldots, N) ; i$ is the diversity branch index; $N$ is the number of combined branches; $r_{i}$ is the $i$ th branch fading amplitude; and $E_{S} / N_{0}$ is the symbol energy-to-Gaussian noise spectral density ratio. The instantenous SNR at each input of the combiner $\gamma_{1}, \ldots, \gamma_{N}$ are gamma distributed with average SNR $\bar{\gamma}_{1}, \ldots, \bar{\gamma}_{N}$ and fading parameters $m_{1}, \ldots, m_{N}$. The correlation coefficient between $\gamma_{p}$ and $\gamma_{q}$ is $\rho_{p q}$ subject to condition (9).

Using the unified approach to evaluate the performance of diversity systems over fading channels proposed by [17], we can obtain the ASEP for coherent detection and the outage probability by using the MGF expressions for correlated Nakagami- $m$ fading with arbitrary shape parameters.

Note that the stability of the integrals used in the evaluation of the ASEP for different modulation schemes given by $[17$, p. 316] is accomplished if all the eigenvalues of the $\mathbf{K}$ matrix given by 


$$
\mathbf{K}=\left[\begin{array}{cccc}
1 & \sqrt{k_{12}} & \cdots & \sqrt{k_{1 N}} \\
\sqrt{k_{12}} & 1 & \cdots & \sqrt{k_{2 N}} \\
\vdots & \vdots & \vdots & \vdots \\
\sqrt{k_{1 N}} & \sqrt{k_{2 N}} & \cdots & 1
\end{array}\right]_{N \times N}
$$

are non-negative. This condition implies that $\mathbf{K}$ is positive definite.

Using the MGF of the SNR at the MRC, the variance of the SNR at the output of the combiner can be easily calculated as

$$
\operatorname{var}(\gamma)=\sum_{i=1}^{N} \frac{\bar{\gamma}_{i}^{2}}{m_{i}}+2 \cdot \sum_{i=1}^{N} \sum_{\substack{l=1 \\ i<l}}^{N} \frac{\bar{\gamma}_{i} \cdot \bar{\gamma}_{l} \cdot \rho_{i l}}{\sqrt{m_{i} \cdot m_{l}}}
$$

with $\bar{\gamma}_{1}, \ldots, \bar{\gamma}_{N}$ being the mean SNR at each branch of the combiner.

\section{NUMERICAL RESULTS}

Numerical results for the ASEP and the probability of outage are given and discussed in this section using the procedure presented above.

In the simulations, we have assumed an exponentially decaying model for the average SNR at each input of the combiner given as

$$
\bar{\gamma}_{n}=\bar{\gamma}_{1} e^{-\frac{(n-1)}{\delta}}, \quad n=1, \ldots, N
$$

and, also an exponential model for the fading parameters written as

$$
m_{n}=m_{1} e^{-\frac{(n-1)}{\eta}}, \quad n=1, \ldots, N
$$

This model has been proposed for the power delay profile in an indoor mobile channel [18], [19].

An exponential correlation model has been assumed in the simulations, where $\rho_{p q}=\rho^{|p-q|} \quad p, q=1, \ldots, N$.

Therefore, the ASEP, the variance of the SNR at the output of the combiner, and the outage probability over correlated Nakagami- $m$ fading can be evaluated using $\Omega_{1}, \ldots, \Omega_{N}$ instead of $\bar{\gamma}_{1}, \ldots, \bar{\gamma}_{N}$ in $(11)$ and $(7)$. 
Fig. 1 shows the effect of the correlation on the average bit error probability with binary frequency shift keying (BFSK) modulation. We have used the following parameters in this evaluation: $m_{1}=2.3, \eta=6, \delta=8$, and $N=2,4$ branches. For instance, the diversity gain (defined as the difference between the average SNR using a diversity technique and the average SNR without the combining required for a specified ASEP) for $10^{-3}$ and $N=2$ branches is: 5.8 $\mathrm{dB}$ and $4.4 \mathrm{~dB}$ for $\rho$ equal to 0 and 0.6 , respectively. Meanwhile, the diversity gain for $10^{-3}$ and $N=4$ branches is: $9.7 \mathrm{~dB}$ and $7.5 \mathrm{~dB}$ for $\rho=0$ and 0.6 , respectively.

Finally, Fig. 2 shows the outage probability as a function of the average SNR of the first contribution, $\bar{\gamma}_{1}$, divided by the SNR protection ratio, $\gamma_{t h}$, for a high number of branches $N=10$. The effect of the unbalance of the average SNR at each input of the combiner is analyzed in this figure. A higher performance degradation is achieved for a lower decay factor $\delta$. However, this effect is more pronounced for a lower fading parameter of the first contribution $m_{1}\left(m_{1}=3.3\right)$.

\section{CONCLUSIONS}

An analytical expression for the MGF of the sum of Nakagami- $m$ correlated variables has been obtained for the analysis of performance metrics of a MRC. From this MGF, we have evaluated the following: the average probability of error and the ASEP for coherent modulations; the variance of the SNR at the combiner output; and the outage probability. This letter also presents the effect of the correlation on an exponential correlation model; the number of combiner branches; and the decay factor in an exponentially decaying model for the fading parameter. The effect of considering equal fading parameters can introduce significant errors in the performance metrics. Additionally, the MGF of the multivariate gamma distribution with arbitrary fading parameters and correlation matrix derived in this letter can be used to evaluate performance parameters in other combiner strategies such as the generalized selection combining technique. 


\section{REFERENCES}

[1] M. Nakagami, "The $m$-distribution-A general formula of intensity distribution of rapid fading," in Statistical Methods of Radio Wave Propagation, W. G. Hoffman Ed., Pergamon Press, Oxford, UK, 1960.

[2] V. A. Aalo, "Performance of maximal-ratio diversity systems in a correlated Nakagami- fading environment," IEEE Trans. Commun., vol. 43, pp. 2360-2369, Aug. 1995.

[3] C. Mun, C-H. Kang, and H-K. Park, "Approximation of SNR statistics for MRC diversity systems in arbitrarily correlated Nakagami fading channels," Electron. Letters, vol. 35, pp. 266-267, Feb. 1999.

[4] Q. T. Zhang, "Maximal-ratio combining over Nakagami fading channels with an arbitrary branch covariance matrix," IEEE Trans. Veh. Technol., vol. 48, pp. 1141 - 1150, Jul. 1999.

[5] M.-S. Alouini, A. Abdi, and M. Kaveh, "Sum of gamma variates and performance of wireless communication systems over Nakagami-fading channels," IEEE Trans. Veh. Technol., vol. 50, pp. 1471-1480, Nov. 2001.

[6] M. Z. Win and J. H. Winters, "On maximal ratio combining in correlated Nakagami channels with unequal fading parameters and SNRs among branches: an analytical framework," in Proc. IEEE Wireless Communications and Networking Conference WCNC'99, vol. 3, Sept. 1999, pp. 1058-1064.

[7] V. A. Aalo, T. Piboongungon, and G. P. Efthymoglou, "Another look at the performance of MRC schemes in Nakagami-m fading channels with arbitrary parameters," IEEE Trans. Commun., vol. 53, pp. 2002-2005, Dec. 2005.

[8] M. W. Gifford, M. Z. Win, and M. Chiani, "Diversity with practical channel estimation," IEEE Trans. Wireless Commun., vol. 4, pp. 1935-1947, July 2005.

[9] J. Cheng and T. Berger, "Capacity and performance analysis for Hybrid Selection/Maximal-Ratio Combining in Nakagami fading with unequal fading parameters and branch powers," in Proc. IEEE International Conference on Communications ICC '03, vol. 5, May 2003, pp. 3031 - 3035. 
[10] M. Z. Win and J. H. Winters, "Analysis of hybrid selection/maximal ratio combining in Rayleigh fading," IEEE Trans. Commun., vol. 47, pp. 1773-1776, Dec. 1999.

[11] M. Z. Win and J. H. Winters, "Virtual branch analysis of symbol error probability for hybrid selection/maximal-ratio combining," IEEE Trans. Commun., vol. 49, pp. 1926-1934, Nov. 2001.

[12] G. P. Efthymoglou, T. Piboongungon, and V. A. Aalo, "Performance of DSCDMA receivers with MRC in Nakagami- $m$ fading channels with arbitrary fading parameters," IEEE Trans. Veh. Technol., vol. 55, pp. 104-114, Jan. 2006.

[13] A. S. Krishnamoorthy and M. Parthasarathy, "A multivariate gamma-type distribution," Annals of Math. Statistics, vol. 22, pp. 549-557, 1951.

[14] J. Reig, L. Rubio, and N. Cardona, "Bivariate Nakagami- $m$ distribution with arbitrary fading parameters," Electron. Letters, vol. 38, pp. 1715-1717, Dec. 2002.

[15] L. Rubio, N. Cardona, L. Juan-Llácer, and J. Reig, "A semi-deterministic propagation model based on a digital map information for the prediction of the coverage area and fading statistics in urban environments," in Proc. Wireless Personal Multimedia Communications WPMC'98, Yokosuka, Japan, 1998, pp. $380-384$.

[16] J. G. Proakis, Digital Communications. New York, NY: McGraw-Hill, 2nd ed., 1989.

[17] M. K. Simon and M.-S. Alouini, Digital Communication over Fading Channels, 2nd ed. New York: Wiley, 2005.

[18] D. Cassioli, M. Z. Win, and A. F. Molisch, "The ultra-wide bandwidth indoor channel: from statistical model to simulations," IEEE J. Select. Areas Commun., vol. 20, pp. 1247-1257, Aug. 2002.

[19] A. Giorgetti, M. Chiani, and M. Z. Win, "The effect of narrowband interference on wideband wireless communications systems," IEEE Trans. Commun., vol. 53, pp. 2139-2149, Dec. 2005. 


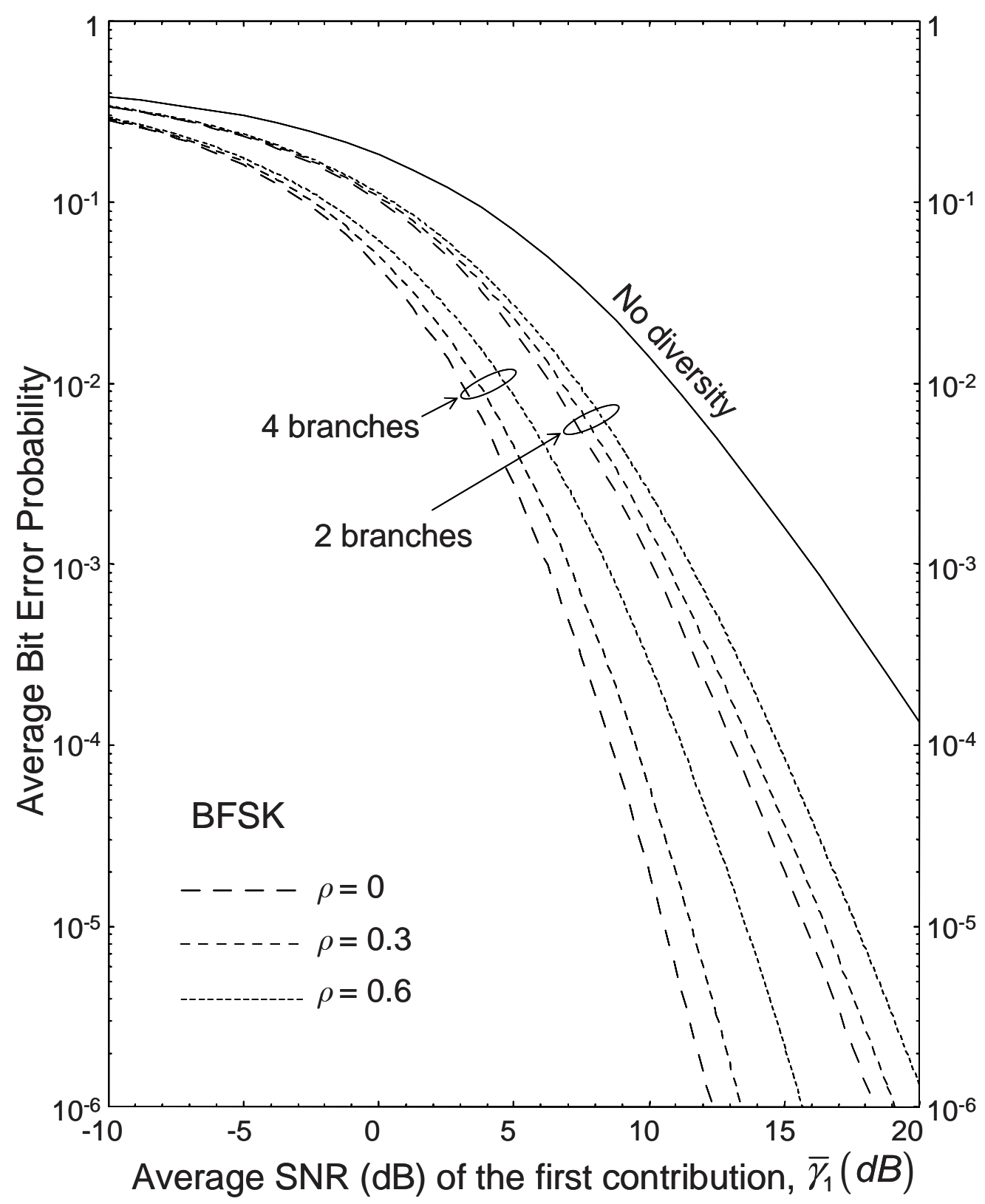

Fig. 1. Average bit error probability versus average SNR of the first contribution for a MRC using BFSK modulation over a correlated Nakagami- $m$ channel in an exponential correlation model. Parameters: $m_{1}=2.3, \eta=6$ and $\delta=8$. 


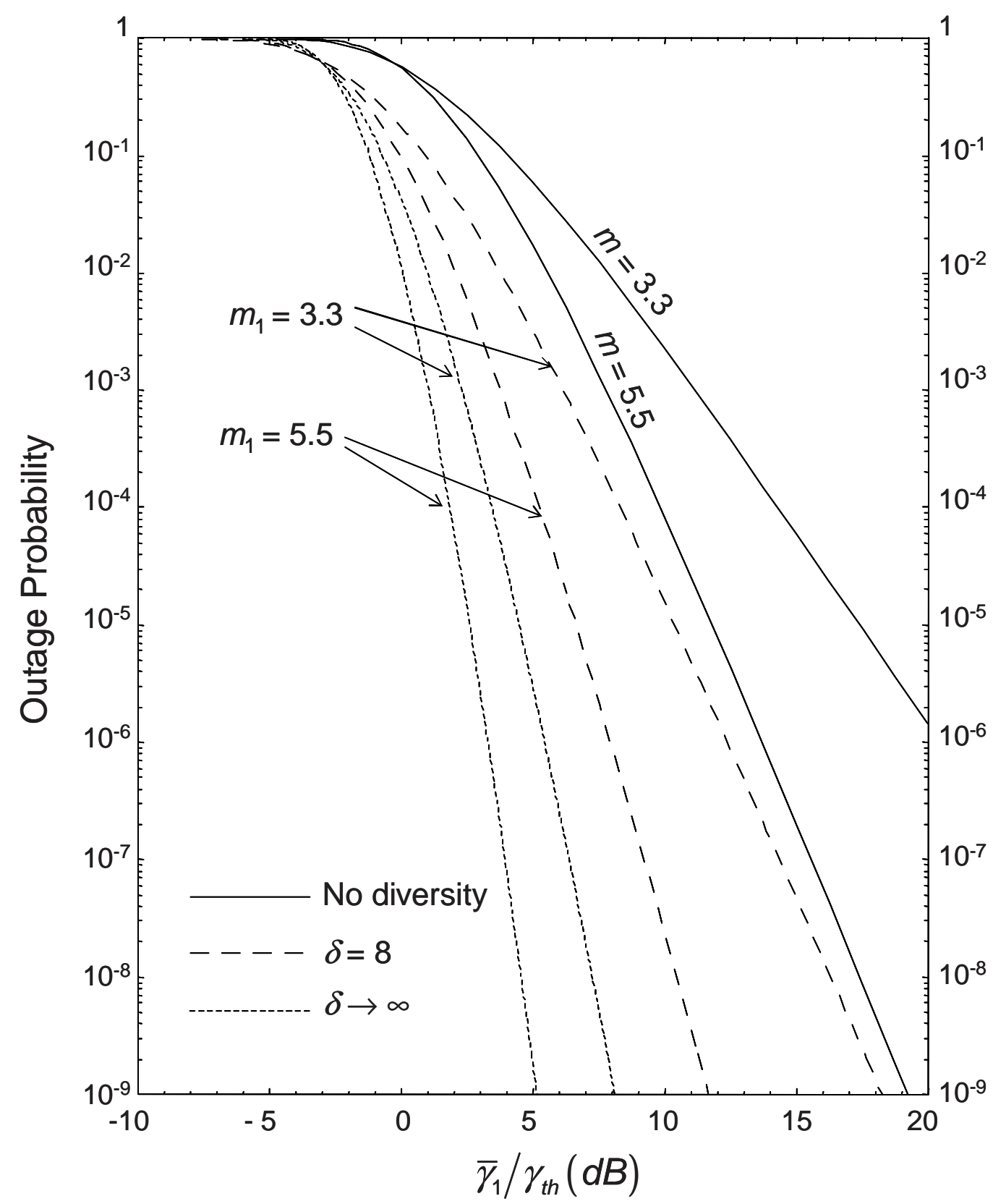

Fig. 2. Outage probability versus average SNR of the first contribution divided by the SNR protection ratio for a MRC over a correlated Nakagami- $m$ channel in an exponential correlation model. Parameters: $N=10, \rho=0.25$ and $\eta=10$. 